\title{
Correction method for obtaining the variationally best ground-state pair density
}

\author{
Masahiko Higuchi \\ Department of Physics, Faculty of Science, Shinshu University, Matsumoto 390-8621, Japan \\ Katsuhiko Higuchi \\ Graduate School of Advanced Sciences of Matter, Hiroshima University, Higashi-Hiroshima 739-8527, Japan
}

(Received 1 May 2011; published 10 October 2011)

\begin{abstract}
We present a correction method for the pair density (PD) to get close to the ground-state one. The PD is corrected to be a variationally best PD within the search region that is extended by adding the uniformly scaled PDs to its elements. The corrected PD is kept $N$-representable and satisfies the virial relation rigorously. The validity of the present method is confirmed by numerical calculations of neon atom. It is shown that the root-mean-square error of the electron-electron interaction and external potential energies, which is a good benchmark for the error of the $\mathrm{PD}$, is reduced by $69.7 \%$ without additional heavy calculations.
\end{abstract}

DOI: 10.1103/PhysRevA.84.044502

PACS number(s): 31.15.ve, 31.15.xt

Electron correlation is one of the main topics in the fields of atomic, molecular, and condensed matter physics. The physical quantity that directly expresses the electron correlation is the diagonal element of the second-order reduced density matrix, which is called the pair density (PD). The PD gives not only the electron density but also expectation values of arbitrary twoparticle operators [1-3]. Therefore, the PD functional theory (PDFT) [4-33] is one of the promising first-principle methods for describing the electron correlation beyond the conventional density functional theory $[34,35]$.

There exist two kinds of well-known problems in developing the PDFT. One is to have to develop the approximate form of the kinetic energy functional since the kinetic energy cannot be expressed by the PD alone [1-3,30-33]. Some approximation is needed for the kinetic energy functional of the PDFT [19,30-33]. We have developed approximate functionals by imposing sum rules for the kinetic energy functional as the restrictive conditions, although they have some room for improvement [19]. The other problem is related to the $N$-representability problem of the PD [1-3,20-29]. Although we have to search the ground-state PD within the set of the $N$-representable PDs [second Hohenberg-Kohn (HK) theorem of the PDFT], the necessary and sufficient conditions for the $N$-representability of the PD have not yet been known in a practical form [1-3,20-29]. We have recently proposed the effective initial theory $[16,18]$ where the search region of PDs is guaranteed to be within a set of $N$-representable PDs but is restricted to the set of PDs that are constructed from single Slater determinants (SDs). Furthermore, we have attempted to extend the variational search region of PDs [17,19] beyond that of the effective initial theory [16,18]. However, it has been eagerly anticipated that such a search region is further extended with keeping the $N$-representability of the PD.

The work described here is motivated by a desire to overcome the above-mentioned well-known problems. In this Brief Report we present a correction method for the PD to get close to the ground-state one, and we show via numerical calculations that this method successfully reduces errors of the PD drastically. The present method is conceptually different from the conventional one [19], and would also have extensive application in the fields such as wave function theory, other types of the density functional schemes, etc.
Let us explain the present correction method in the following three steps (i)-(iii).

(i) As the first step, we shall show that the kinetic energy functional which is consistent with the scaling property satisfies the virial relation exactly. Here suppose that the search region of PDs fully covers a set of the $N$-representable PDs. Applying the scalings of the electron coordinates, the PD $\gamma^{(2)}\left(\mathbf{r} \mathbf{r}^{\prime} ; \mathbf{r r}^{\prime}\right)$ is transformed into

$$
\gamma_{\lambda}^{(2)}\left(\mathbf{r} \mathbf{r}^{\prime} ; \mathbf{r} \mathbf{r}^{\prime}\right)=\lambda^{6} \gamma^{(2)}\left(\lambda \mathbf{r} \lambda \mathbf{r}^{\prime} ; \lambda \mathbf{r} \lambda \mathbf{r}^{\prime}\right)
$$

where $\lambda$ is the scaling parameter. $\gamma_{\lambda}^{(2)}\left(\mathbf{r r}^{\prime} ; \mathbf{r r}^{\prime}\right)$ is the PD scaled from $\gamma^{(2)}\left(\mathbf{r r}^{\prime} ; \mathbf{r r}^{\prime}\right)$, which is called the scaled PD. The kinetic energy functional that is consistent with the scaling property satisfies the following relation $[8,16,19]$ :

$$
T\left[\gamma_{\lambda}^{(2)}\right]=\lambda^{2} T\left[\gamma^{(2)}\right]
$$

Similarly to Eq. (2), the electron-electron interaction and external potential energies to the scaled PD are, respectively, given by

$$
W\left[\gamma_{\lambda}^{(2)}\right]=\lambda W\left[\gamma^{(2)}\right]
$$

and

$$
V\left[\gamma_{\lambda}^{(2)}\right]=\frac{2}{N-1} \iint v_{\text {ext }}\left(\frac{\mathbf{r}}{\lambda}\right) \gamma^{(2)}\left(\mathbf{r r}^{\prime} ; \mathbf{r r}^{\prime}\right) d \mathbf{r} d \mathbf{r}^{\prime},
$$

where $v_{\text {ext }}(\mathbf{r})$ denotes the external potential and where $N$ is the number of electrons. Due to the second HK theorem of the PDFT, the total energy functional $E\left[\gamma^{(2)}\right]\left(=T\left[\gamma^{(2)}\right]+W\left[\gamma^{(2)}\right]+V\left[\gamma^{(2)}\right]\right)$ is minimum at $\gamma_{0}^{(2)}$ that corresponds to the variationally best PD among all of the $N$-representable PDs. Using the scaled PD, the theorem can be written as $d E\left[\gamma_{0, \lambda}^{(2)}\right] /\left.d \lambda\right|_{\lambda=1}=0$. Here $\gamma_{0, \lambda}^{(2)}$ is the PD scaled from $\gamma_{0}^{(2)}$, and is defined similarly to Eq. (1). Substituting Eqs. (2), (3), and (4) into this theorem, we have

$$
2 T\left[\gamma_{0}^{(2)}\right]+W\left[\gamma_{0}^{(2)}\right]=\iint \frac{\left[\mathbf{r} \cdot \nabla v_{\mathrm{ext}}(\mathbf{r})\right]}{N-1} \gamma_{0}^{(2)}\left(\mathbf{r} \mathbf{r}^{\prime} ; \mathbf{r r}^{\prime}\right) d \mathbf{r} d \mathbf{r}^{\prime}
$$

Equation (5) is the virial relation of the PDFT. It should be noted that even though the kinetic energy functional that satisfies Eq. (2) is not an exact but an approximate form, Eq. (5) 


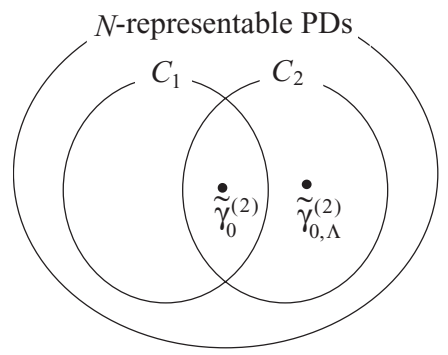

FIG. 1. The relations between various sets of PDs. The set $C_{1}$ covers a set of the $N$-representable PDs not fully but partially. The set $C_{2}$ consists of the uniformly scaled PDs. The variationally best PD within $C_{1}$, and that within $C_{1} \cup C_{2}$, are denoted by $\tilde{\gamma}_{0}^{(2)}$ and $\tilde{\gamma}_{0, \Lambda}^{(2)}$, respectively.

exactly holds if the search region fully covers the set of the $N$-representable PDs.

(ii) Next, we shall consider the case where the search region not fully but partially covers a set of the $N$-representable PDs. Suppose that such a partial search region is denoted as $C_{1}$ (see Fig. 1), and that the total energy functional is minimum at $\tilde{\gamma}_{0}^{(2)}$ that corresponds to the variationally best PD within the set $C_{1}$. Due to the incomplete cover of $N$-representable PDs by the set $C_{1}$, it could be that not all of the scaled PDs $\tilde{\gamma}_{0, \lambda}^{(2)}$ are included in the set $C_{1}$. We denote the set of $\tilde{\gamma}_{0, \lambda}^{(2)}$ as $C_{2}$ (Fig. 1). In this case, the total energy functional $E\left[\tilde{\gamma}_{0, \lambda}^{(2)}\right]$ does not always take the minimum at $\lambda=1$. Namely, we have $d E\left[\tilde{\gamma}_{0, \lambda}^{(2)}\right] /\left.d \lambda\right|_{\lambda=1} \neq 0$. Like the derivation of Eq. (5), this inequality leads to the relation such that the left-hand side of Eq. (5) is not equal to the right-hand side. Thus, if the search region does not fully cover the set of the $N$-representable PDs, the virial relation does not hold even though the kinetic energy functional satisfies Eq. (2). This fact can be used as a criterion of whether the search region fully covers the set of the $N$-representable PDs or not. Here let us define the virial ratio which indicates to what extent the virial relation holds:

$$
R_{v}=\frac{W\left[\tilde{\gamma}_{0}^{(2)}\right]-\iint \frac{\left[\mathbf{r} \cdot \nabla v_{\text {ext }}(\mathbf{r})\right]}{N-1} \tilde{\gamma}_{0}^{(2)}\left(\mathbf{r r}^{\prime} ; \mathbf{r r}^{\prime}\right) d \mathbf{r} d \mathbf{r}^{\prime}}{T\left[\tilde{\gamma}_{0}^{(2)}\right]} .
$$

Using this ratio, the above-mentioned criterion can be restated as follows: the deviation of $R_{v}$ from the value -2.0 means the insufficiency of the search region of PDs if the kinetic energy functional satisfies Eq. (2) [36].

(iii) When $R_{v}$ deviates from -2.0 , a correction method that is related to the extension of the search region is desired. The key point of the present correction method is the extension of the search region by adding the scaled PDs to the search region. Figure 1 truly shows the relation between the original search region $C_{1}$ and newly added search region $C_{2}$. Both sets $C_{1}$ and $C_{2}$ are subsets of the set of the $N$-representable PDs. The above-mentioned inequality $d E\left[\tilde{\gamma}_{0, \lambda}^{(2)}\right] /\left.d \lambda\right|_{\lambda=1} \neq 0$ means that the set $C_{2}$ could possibly include the PD that takes a total energy lower than what the best PD within the set $C_{1}$ (i.e., $\tilde{\gamma}_{0}^{(2)}$ ) takes. Such a PD exists in $C_{2} \cap \bar{C}_{1}$ and is denoted as $\tilde{\gamma}_{0, \Lambda}^{(2)}$, where $\bar{C}_{1}$ is the complementary set of $C_{1}$ (Fig. 1). Then the inequality $d E\left[\tilde{\gamma}_{0, \lambda}^{(2)}\right] /\left.d \lambda\right|_{\lambda=1} \neq 0$ is rewritten as $d E\left[\tilde{\gamma}_{0, \lambda}^{(2)}\right] /\left.d \lambda\right|_{\lambda=\Lambda}=0$. Substituting Eqs. (2), (3), and (4) into this equation, we obtain

$$
\begin{aligned}
& 2 \Lambda T\left[\tilde{\gamma}_{0}^{(2)}\right]+W\left[\tilde{\gamma}_{0}^{(2)}\right] \\
& \quad=\iint \frac{\left[\mathbf{r} \cdot \nabla_{\frac{\mathrm{r}}{\Lambda}} v_{\mathrm{ext}}\left(\frac{\mathbf{r}}{\Lambda}\right)\right]}{\Lambda^{2}(N-1)} \tilde{\gamma}_{0}^{(2)}\left(\mathbf{r r}^{\prime} ; \mathbf{r r}^{\prime}\right) d \mathbf{r} d \mathbf{r}^{\prime} .
\end{aligned}
$$

If we consider the isolated atomic system, Eq. (7) is easily rewritten as

$$
2 \Lambda T\left[\tilde{\gamma}_{0}^{(2)}\right]+W\left[\tilde{\gamma}_{0}^{(2)}\right]=-V\left[\tilde{\gamma}_{0}^{(2)}\right]
$$

From Eq. (7) or (8) we can determine the value of $\Lambda$. The scaled PD $\tilde{\gamma}_{0, \Lambda}^{(2)}$ with $\Lambda$ thus determined is the variationally best one within the union of two sets, that is, $C_{1} \cup C_{2}$. That is to say, a correction of the ground-state PD from $\tilde{\gamma}_{0}^{(2)}$ to $\tilde{\gamma}_{0, \Lambda}^{(2)}$ is accomplished by extending the search region from $C_{1}$ to $C_{1} \cup C_{2}$. It is obvious that the corrected $\mathrm{PD} \tilde{\gamma}_{0, \Lambda}^{(2)}$ remains $N$-representable. Furthermore, it is easily confirmed that the virial relation exactly holds for the corrected $\mathrm{PD} \tilde{\gamma}_{0, \Lambda}^{(2)}$. Namely, the correction method can supplement the search region of PDs with $C_{2}$ to make the resultant PD $\tilde{\gamma}_{0, \Lambda}^{(2)}$ satisfy the virial theorem [37].

The procedure of this correction method is as follows. First we prepare the approximate form of the kinetic energy functional that satisfies Eq. (2), and determine the search region of PDs. Using this kinetic energy functional, we calculate $\tilde{\gamma}_{0}^{(2)}$. Substituting $\tilde{\gamma}_{0}^{(2)}$ into Eq. (7) or (8), the scaling parameter $\Lambda$ is obtained. This $\Lambda$ immediately leads to the corrected PD $\tilde{\gamma}_{0, \Lambda}^{(2)}$ by using Eq. (1). Of course, the kinetic energy, electron-electron interaction energy and external potential energy for $\tilde{\gamma}_{0, \Lambda}^{(2)}$ can be easily calculated from Eqs. (2), (3), and (4), respectively. One of striking points of this correction method is that the heavy calculation task is not needed for the corrections of the PD and related quantities.

In order to check the validity of the present method, we perform numerical calculations for a neutral neon atom. As the approximate form of the kinetic energy functional that satisfies Eq. (2), we adopt the following functional [38]:

$$
\begin{aligned}
T\left[\gamma^{(2)}\right]= & \iint\left\{K\left(\frac{1}{r^{2}}+\frac{1}{r^{\prime 2}}\right)+K^{\prime}\left[\frac{\cos \theta}{2 r^{2}} \ln \left(\frac{r}{r^{\prime}}\right)\right.\right. \\
& \left.\left.+\frac{\cos \theta^{\prime}}{2 r^{\prime 2}} \ln \left(\frac{r^{\prime}}{r}\right)\right]\right\} \gamma^{(2)}\left(\mathbf{r r}^{\prime} ; \mathbf{r r}^{\prime}\right) d \mathbf{r} d \mathbf{r}^{\prime},
\end{aligned}
$$

where $K$ and $K^{\prime}$ are arbitrary constants that are determined later. The computational PD functional scheme [19] is utilized so as to obtain $\tilde{\gamma}_{0}^{(2)}$. According to this scheme, we adopt as the search region the set of PDs that are calculated from the correlated wave functions. As the correlated wave functions, we take the linear combination of the ground-state and doubly excited SDs that consist of eigenfunctions of the effective initial scheme $[16,18]$. Among all doubly excited SDs, we choose the SDs that have nonnegligible contributions to the resultant PD. A total of 2861 SDs and 12275 SDs, the constituent eigenfunctions of which have the principal quantum number up to 6 and 11, respectively, are chosen as basis functions in constructing PDs. The details of preparing the set of eigenfunctions are given in previous papers [16,19]. 
As the set $C_{1}$, we take the set of PDs that are constructed by 2861 SDs. First, by means of the computational PD functional scheme [19], we calculate $\tilde{\gamma}_{0}^{(2)}$ that is the variationally best PD within the set $C_{1}$. Then the corrected $\mathrm{PD} \tilde{\gamma}_{0, \Lambda}^{(2)}$ is calculated by using Eqs. (1) and (8). We also prepare the search region that is extended with increasing the number of basis SDs (NSD) from 2861 to 12275 . Hereafter, we denote this search region of PDs as $C_{1}^{\prime}$. In order to show that the present extension method of the search region of PDs is more efficient than the conventional extension method, the variationally best PD within the set $C_{1}^{\prime}$ is also calculated by means of the computational PD functional scheme [19].

Using $\tilde{\gamma}_{0}^{(\dot{ })}$ and $\tilde{\gamma}_{0, \Lambda}^{(2)}$, we calculate errors of the electronelectron interaction energy, external potential energy, kinetic energy, and virial ratio that are denoted by $\Delta W, \Delta V$, $\Delta T$, and $\Delta R_{v}$, respectively. As the reference data of these energies, we adopt the results of the configuration interaction method $[39,40]$. The root-mean-square error (RMSE) of the electron-electron interaction and external potential energies is also calculated by $\sqrt{\left[(\Delta W)^{2}+(\Delta V)^{2}\right] / 2}$. The RMSE is considered as a good benchmark for to what extent the resultant PD is close to the correct ground-state PD. This is because accuracies of both the electron-electron interaction and external potential energies are dependent only on that of the PD.

The above-mentioned calculations are performed with changing the values of $K$ and $K^{\prime}$ that appear in Eq. (9). These values are determined by requiring the approximate functional to have two desirable features. One is that the approximate functional reproduces the Hartree-Fock kinetic energy if the PD coincides with the Hartree-Fock PD. The other is that the RMSE of the electron-electron interaction and external potential energies is minimized with respect to parameters. This determination process is implemented in individual cases before and after the correction.

Calculation results are shown in Fig. 2. From this figure we find the following points.

(1) It is found in Fig. 2 that the present method reduces $\Delta W$ and $\Delta V$ by $89.7 \%$ and $66.3 \%$, respectively (reductions from case $a$ to $c$ for " $\Delta W$ " and " $\Delta V$," respectively, in

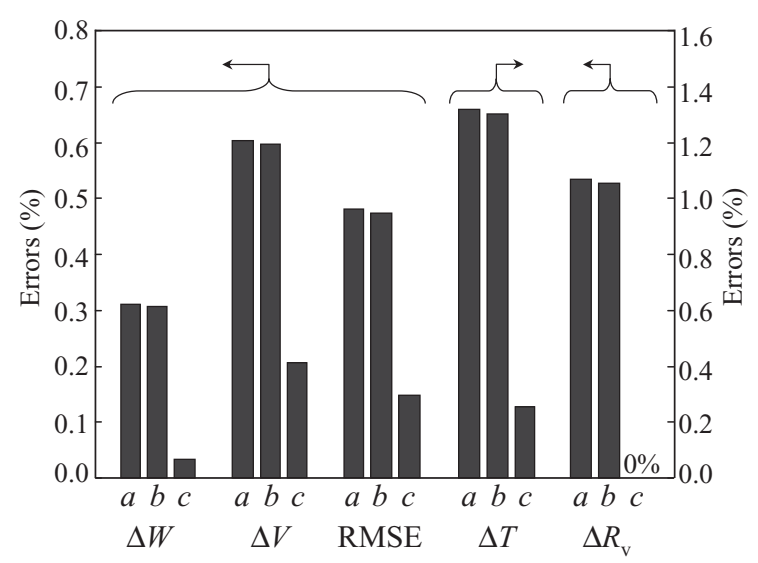

FIG. 2. The calculation results of the $\Delta W, \Delta V$, RMSE, $\Delta T$, and $\Delta R_{v}$. The search region $C_{1}, C_{1}^{\prime}$, and $C_{1} \cup C_{2}$ are simply denoted by the symbols $a, b$, and $c$, respectively.
Fig. 2). On the other hand, only $1.3 \%$ and $1.1 \%$ reductions are made via increasing the NSD from 2861 to 12275 in $\Delta W$ and $\Delta V$, respectively (reduction from case $a$ to $b$ for " $\Delta W$ " and " $\Delta V$ ", respectively). Also, as shown in Fig. 2, the RMSE is remarkably reduced by $69.7 \%$ (reduction from case $a$ to $c$ ), while it is improved only $1.2 \%$ by increasing the NSD (reduction from case $a$ to $b$ ). Thus the reduction rate of the present method is much larger than that of the extension method with increasing the NSD. This leads to that the search region of PDs is more effectively extended by adding the uniformly scaled PDs than by increasing the NSD. Furthermore, it should be noticed that the present method is feasible without additional heavy calculations.

(2) It is found in Fig. 2 that $\Delta T$ is also reduced much more effectively by the present method than by increasing the NSD (80.9\% and $1.2 \%$ reductions for the former and latter, respectively). In general, the accuracy of the kinetic energy is dependent on both the appropriateness of the approximate form of the functional and that of the resultant PD. Judging from the results of the RMSE, $\Delta W$, and $\Delta V$ (Fig. 2), the resultant PD seems to be improved to be close to the ground state one. Therefore we can deduce that the approximate form itself, which is given by Eq. (9), would also be sound, though we need to apply Eq. (9) to the other systems in order to further ensure its soundness [38].

(3) It is found in Fig. 2 that the virial ratios $R_{v}$ before the correction deviate from the correct value -2.0 . Using the criterion on the search region, these deviations mean that not only the search region $C_{1}$ but also the search region $C_{1}^{\prime}$ are insufficient since the approximate functional Eq. (9) is consistent with Eq. (2). That is to say, even though more than 12000 SDs are used in constructing PDs, the search region is not extended effectively. On the other hand, $R_{v}$ after the correction is rigorously equal to -2.0 , as it should be. These tendencies seem to be consistent with those mentioned in (1) and (2).

Thus, the present method improves not only $\Delta W, \Delta V$, and RMSE but also $\Delta T$ and $\Delta R_{v}$ quite substantially and effectively. We can therefore say that the PD is corrected to be close to the ground-state one appropriately via the present method.

In conclusion, we summarize the features of the correction method proposed here. The most distinctive feature is that

(1) the search region is extended by adding a set of the scaled PDs to elements of the search region.

The resultant PD that is corrected by this method possesses the following features:

(2) the corrected PD is $N$-representable,

(3) the corrected PD satisfies the virial relation exactly.

The validity of the present method is successfully confirmed by numerical calculations of neon atomic system. It is shown that

(4) not only the RMSE but also errors of the kinetic energy, electron-electron interaction energy, and external potential energy are individually all reduced definitely,

(5) the correction is effective enough even in the small size calculations.

Due to the computational easiness of the correction, this method will be applicable to larger systems such as molecules, clusters, and solids. 
[1] R. G. Parr and W. Yang, Density-Functional Theory of Atoms and Molecules, Chap. 2 (Oxford University Press, New York, 1989).

[2] A. J. Coleman, Rev. Mod. Phys. 35, 668 (1963).

[3] J. Cioslowski, ed., Many-Electron Densities and Reduced Density Matrices (Kluwer, Dordrecht, The Netherlands, 2000).

[4] P. Ziesche, Phys. Lett. A 195, 213 (1994).

[5] P. Ziesche, Int. J. Quantum Chem. 60, 1361 (1996).

[6] A. Gonis, T. C. Schulthess, J. van Ek, and P. E. A. Turchi, Phys. Rev. Lett. 77, 2981 (1996).

[7] A. Gonis, T. C. Schulthess, P. E. A. Turchi, and J. van Ek, Phys. Rev. B 56, 9335 (1997)

[8] M. Levy and P. Ziesche, J. Chem. Phys. 115, 9110 (2001).

[9] F. Furche, Phys. Rev. A 70, 022514 (2004).

[10] Á. Nagy, Phys. Rev. A 66, 022505 (2002).

[11] Á. Nagy and C. Amovilli, J. Chem. Phys. 121, 6640 (2004).

[12] B. Hetényi, L. Brualla, and S. Fantoni, Phys. Rev. Lett. 93, 170202 (2004).

[13] P. W. Ayers and M. Levy, J. Chem. Sci. 117, 507 (2005).

[14] Á. Nagy, Int. J. Quantum Chem. 106, 1043 (2006).

[15] P. W. Ayers, S. Golden, and M. Levy, J. Chem. Phys. 124, 054101 (2006).

[16] M. Higuchi and K. Higuchi, Physica B 387, 117 (2007).

[17] M. Higuchi and K. Higuchi, Phys. Rev. A 75, 042510 (2007).

[18] M. Higuchi and K. Higuchi, Phys. Rev. B 78, 125101 (2008).

[19] K. Higuchi and M. Higuchi, Phys. Rev. B 82, 155135 (2010).

[20] E. R. Davidson, Chem. Phys. Lett. 246, 209 (1995).

[21] S. Kh. Samvelyan, Int. J. Quantum Chem. 65, 127 (1997).

[22] M.-E. Pistol, Chem. Phys. Lett. 400, 548 (2004).

[23] P. Gori-Giorgi and A. Savin, Philos. Mag. 86, 2643 (2006).

[24] M.-E. Pistol, Chem. Phys. Lett. 422, 363 (2006).
[25] M.-E. Pistol, Chem. Phys. Lett. 431, 216 (2006).

[26] P. W. Ayers and S. Liu, Phys. Rev. A 75, 022514 (2007).

[27] P. W. Ayers and E. R. Davidson, Adv. Chem. Phys. 134, 443 (2007).

[28] M.-E. Pistol, Chem. Phys. Lett. 449, 208 (2007).

[29] B. Hetényi and A. W. Hauser, Phys. Rev. B 77, 155110 (2008).

[30] N. H. March and R. Santamaria, Int. J. Quantum Chem. 39, 585 (1991).

[31] P. W. Ayers, J. Math. Phys. 46, 062107 (2005).

[32] P. W. Ayers and M. Levy, Chem. Phys. Lett. 415, 211 (2005).

[33] R. Cuevas-Saavedra and P. W. Ayers, Int. J. Quantum Chem. 109, 1699 (2009).

[34] P. Hohenberg and W. Kohn, Phys. Rev. 136, B864 (1964).

[35] W. Kohn and L. J. Sham, Phys. Rev. 140, A1133 (1965).

[36] This criterion corresponds to just the following statement: $R_{v}$ is equal to -2.0 if the search region of PDs covers fully the set of the N-representable PDs. Note that the reverse does not necessarily hold.

[37] Concerning the previous applications of the virial theorem to the reduction of errors, for examples please see the following papers: D. E. Magnoli and J. R. Murdoch, Int. J. Quantum Chem. 22, 1249 (1982); R. J. Tweed and J. Langlois, J. Phys. B 19, 3565 (1986); M. Kodera, K. Higuchi, A. Narita, and M. Higuchi, Phys. Rev. A 78, 012501 (2008).

[38] K. Higuchi and M. Higuchi, unpublished. We propose this approximate form by using the equilibrium condition of "no net external force of single-atom systems" in addition to two restrictive conditions that are employed in Ref. [19].

[39] A. Veillard and E. Clementi, J. Chem. Phys. 49, 2415 (1968).

[40] A. V. Bunge and R. O. Esquivel, Phys. Rev. A 34, 853 (1986). 